\title{
Clinical, Epidemiological and Microbiological Profile of A Potentially Pathogenic Environmental Saprophyte, Burkholderia pseudomallei; at A Tertiary Care Hospital in Coastal India
}

\author{
Sruthi Vinayan (iD and U. Pratibha Bhat* \\ Department of Microbiology, Nitte (Deemed to be University), KS Hegde Medical Academy (KSHEMA), \\ Deralakatte, Mangaluru-575018, Karnataka, India.
}

\begin{abstract}
Melioidosis is a severe systemic infectious disease caused by Burkholderia pseudomallei, a gram-negative bacillus with bipolar staining. It is an environmental saprophyte endemic to Southeast Asia and Northern Australia. The disease can have varying manifestations. This is a retrospective study of the clinical and microbiological profile of culture proven cases of melioidosis who presented to a tertiary care hospital in Coastal Karnataka between January 2018 and December 2020. The epidemiological, demographic, clinical and laboratory characteristics were studied and analyzed. A total of $\mathbf{2 7}$ cases were seen during the study period. All patients were from the western coastal areas of India. Fever was the most common presenting complaint. Analysis of the clinical manifestations showed 11 (40.74\%) with bacteremia. Pneumonia was the most common primary clinical presentation with 11 cases (40.74\%). $9(33.3 \%)$ patients had an abscess in some part of the body on presentation. Secondary foci were seen in $\mathbf{5}(\mathbf{1 8 . 5 \% )}$ patients. The prominent risk factors seen were history of type $\mathbf{2}$ diabetes mellitus, age $>\mathbf{4 0}$ years, alcoholism and smoking. $13(48.15 \%)$ were started with the treatment regimen for melioidosis. Only 8 (29.63\%) were prescribed the eradication treatment regimen. One case which was inadequately treated came back with reactivation of melioidosis. Varied clinical presentation of melioidosis makes the specific clinical diagnosis difficult. Due to the high mortality and morbidity rate, early diagnosis and prompt management is warranted, this requires clinical vigilance and an intensive microbiological workup. Lack of adherence to the treatment protocol can lead to reactivation.
\end{abstract}

Keywords: Melioidosis, pneumonia, therapy, recurrence

*Correspondence: pratibhabhat.u@gmail.com; +91 9482274526

(Received: August 15, 2021; accepted: December 3, 2021)

Citation: Vinayan S, Bhat UP. Clinical, Epidemiological and Microbiological Profile of A Potentially Pathogenic Environmental Saprophyte, Burkholderia pseudomallei; at A Tertiary Care Hospital in Coastal India. J Pure Appl Microbiol. 2022;16(1):193-200. doi: 10.22207/JPAM.16.1.09

(C) The Author(s) 2022. Open Access. This article is distributed under the terms of the Creative Commons Attribution 4.0 International License which permits unrestricted use, sharing, distribution, and reproduction in any medium, provided you give appropriate credit to the original author(s) and the source, provide a link to the Creative Commons license, and indicate if changes were made. 


\section{INTRODUCTION}

Burkholderia pseudomallei was first recognised in 1911 by Whitmore and Krishnaswami in Burma. By 1917, Krishnaswami had reported more than 100 cases, which was $5 \%$ of all the autopsy examinations during that time. In later years, cases of melioidosis were reported in soldiers fighting in Vietnam War. The United States Centres for Disease Control and Prevention (CDC) have classified the organism as an agent of bioterrorism. ${ }^{1,2}$ Burkholderia pseudomallei, which is a gram-negative bacilli with bipolar staining; causes an infectious disease called melioidosis. It is an environmental saprophyte present in the soil and ground water, which can infect humans and a wide range of animals. The organism is endemic to tropical countries, especially in Southeast Asia and Northern Australia. ${ }^{3}$ Infections can occur by exposure through broken skin, inhalation or ingestion. ${ }^{4}$

Melioidosis cases arise more frequently during the monsoon or tropical storms with male sex, diabetes mellitus and alcohol abuse being few risk factors. ${ }^{5}$ The disease manifestations being protean in nature, ranges from localized to multifocal infection with or without septicemia, hence termed as 'the great mimicker' ${ }^{6,7}$ Pulmonary involvement is the most common acute infection in adults, often resembling community-acquired pneumonia and tuberculosis, resulting in frequent misdiagnosis of the condition. ${ }^{8}$ The incubation period varies widely. After exposure to a heavy inoculum, the incubation period may be as short as one day. Cases presenting with symptoms for more than two months to few years are also described. ${ }^{3}$ Recognition of melioidosis based on clinical presentations can be challenging, and a delay in diagnosis can result in fatality.

Antibiotic therapy in 2 phases is important in the treatment of melioidosis. The intensive or acute phase comprises of intravenous (i.v.) ceftazime/imipenem/meropenem for duration of $>=10$ days. This phase aims to prevent patient death by overwhelming sepsis. The eradication phase consists of oral antibiotics like cotrimoxazole or doxycycline or amoxicillin clavulanate for a minimum of 12 weeks. This phase is aimed at targeting the residual bacteria and prevent relapse. $^{9}$
Here, we aim to study the epidemiology, clinical presentations, laboratory characteristics and treatment of culture proven cases of melioidosis which presented to our tertiary care center.

\section{MATERIALS AND METHODS Study setting and design}

This is a retrospective study of culture proven cases of melioidosis who presented to a tertiary care hospital in Mangaluru, located in the coastal district; Dakshina Kannada, Karnataka. Retrospective data was collected over a period of 3 years; between January 2018 and December 2020. Patients from the neighbouring coastal districts of Udupi, Uttara Kannada, Kasargod, Kannur also present to this hospital. These districts receive heavy rains due to Southwest monsoons between June to September with an average rainfall of $900 \mathrm{~mm}$; trailing off to occasional rainfalls with an average of $200 \mathrm{~mm}$ between October to December. ${ }^{10}$ All patients whose clinical specimens received in the microbiology laboratory which yielded growth of Burkholderia pseudomallei were included in the study.

\section{Laboratory technique}

Blood cultures and sterile body fluid cultures were performed using the BacT/ALERT 3D (bioMerieux) system, which is a fully automated blood culture system. Blood was incubated in BacT/Alert FA Plus bottles and for sterile body fluids BacT/Alert PF plus was used. Once the culture bottles flagged positive, it was subcultured onto MacConkey's agar and blood agar. All other specimens were cultured using conventional method of inoculation onto culture media like MacConkey's agar and sheep blood agar and incubated at $37{ }^{\circ} \mathrm{C}$. Identification of isolates were done by standard microbiological techniques that included Gram staining showing gram-negative bacilli with bipolar staining or 'safety pin' appearance, motile, growth on MacConkey's agar showing rough, wrinkled, pink coloured colonies with a metallic sheen, slow oxidase positive, indole negative, lysine decarboxylase negative, arginine dihydrolase positive and resistant to gentamicin $(10 \mathrm{mcg} /$ disc) and colistin (10 mcg/ disc). Additionally, automated identification was also performed using 
Vitek-2 compact system (bioMerieux). Clinical and laboratory Standards Institute (CLSI) M45 guidelines recommends only broth microdilution method for antibiotic susceptibility testing of B.pseudomallei. ${ }^{11}$ This method is cumbersome and was not done during the routine diagnostics. Hence, to guide patient treatment; antibiotic susceptibility testing was done by Vitek-2 compact system (bioMerieux). The system performs and reports identification of B.pseudomallei; but the software database for analysis of the minimum inhibitory concentration (MIC) values for B.pseudomallei is unavailable. Hence, the MIC values were extracted from the system as per the database for B.cepacia complex and then the values were analyzed in comparison to the breakpoints for B.pseudomallei as given in CLSI M45 guidelines and interpreted accordingly. The MIC of 4 antibiotics namely; ceftazidime, imipenem, cotrimoxazole and amoxicillin-clavulanate as determined by the Vitek 2 system was analyzed using CLSI guidelines. ${ }^{11}$ The interpretation was made as follows: for ceftazidime (sensitive <= $8 \mu \mathrm{g} / \mathrm{ml},>=32 \mu \mathrm{g} / \mathrm{ml}$ resistant $)$, imipenem $(<=4 \mu \mathrm{g} /$ $\mathrm{ml}$ sensitive, $>=16 \mu \mathrm{g} / \mathrm{ml}$ resistant), amoxicillin clavulanate $(<=8 / 4 \mu \mathrm{g} / \mathrm{ml}$ sensitive, $>=32 / 16 \mu \mathrm{g} / \mathrm{ml}$ resistant), cotrimoxazole $(<=2 / 38 \mu \mathrm{g} / \mathrm{ml}$ sensitive, $>=4 / 76 \mu \mathrm{g} / \mathrm{ml}$ resistant). CLSI has no breakpoints defined for meropenem; though there are reports of successful use of meropenem in the treatment of melioidosis. ${ }^{9,12}$ Doxycycline susceptibility for the organism was not tested as the panel of antibiotics for nonfermenting gram-negative bacilli in Vitek 2 did not include doxycycline.

The case records of the culture proven cases was retrospectively studied with respect to the following parameters; epidemiological characteristics like geographical location of the patient, month of presentation to the hospital; patient demographics like age and sex, clinical characteristics like history of presenting illness, duration of symptoms, comorbidities, radiological features, laboratory parameters like total leucocyte and differential count, specimen received for culture in the laboratory, microbiological features of the growth, treatment received and outcome. Patient records were checked for treatment with any of the following antibiotics; ceftazidime, meropenem, imipenem, cotrimoxazole, amoxicillin-clavulanate and doxycycline; which have been described as drugs of choice for the treatment of melioidosis. Intensive phase was considered to be completed if the patient had received either ceftazidime/meropenem/ imipenem with or without cotrimoxazole for a minimum duration of 10 days. Eradication phase of treatment was considered as adequate if the patient had been prescribed cotrimoxazole/ amoxicillin-clavulanic acid/doxycycline for a minimum period of 12 weeks. ${ }^{9,13}$ At the study site, for a case of melioidosis the treatment regimen recommended is either i.v. ceftazidime or i.v imipenem/meropenem for a minimum of 2 weeks and oral cotrimoxazole for a minimum of 12 weeks. The data was tabulated and results were analyzed using statistical methods like measures of frequency and percentages.

\section{RESULTS}

A total of 27 culture proven cases of melioidosis were seen during the study period; 9 cases in the year 2018, 7 cases in 2019 and 11 in the year 2020. 19 patients (70.4\%) presented during the latter half of the year (with 10 cases between July to September and 9 cases between October to December); while only 8 (29.6\%) patients presented during the first half (January to June). All patients were from the western coastal areas of India. Patients hailed from coastal districts of Karnataka and Kerala namely, Dakshina Kannada (10 cases), Uttar Kannada (8 cases) Udupi (1), Kannur (5), Kasargod (3).

The age of the patients ranged from 15 years to 63 years with a median of 52 years. $21(77.8 \%)$ of the patients were $>40$ years old; out of which 15 patients were between 5160 years of age. $22(81.4 \%)$ were males and 5 (18.5\%) were females. 16 patients were initially seen at the outpatient department of General medicine, 6 cases presented to the department of General surgery and the rest presented to the department of Orthopaedics, Pulmonary medicine, Otorhinolaryngology, Nephrology and Neurology with one case each respectively.

The duration of history of presenting complaints were as short as 1 day to as long as 6 months. Fever was the most common presenting complaint. 19 (70.4\%) had fever as the chief complaint with 9 patients having fever for a duration of $>1$ month (average 2 months) and 
Vinayan \& Bhat | J Pure Appl Microbiol | 16(1):193-200 | March 2022 | https://doi.org/10.22207/JPAM.16.1.09

Table 1.Primary clinical presentation of culture confirmed melioidosis cases

\begin{tabular}{lccc}
\hline $\begin{array}{l}\text { Primary } \\
\text { diagnosis }\end{array}$ & $\begin{array}{c}\text { Anatomic } \\
\text { location }\end{array}$ & $\begin{array}{c}\text { Number } \\
\text { (percentage) } n=27\end{array}$ & $\begin{array}{c}\text { Number with } \\
\text { bacteremia }\end{array}$ \\
\hline $\begin{array}{l}\text { Pneumonia } \\
\text { Osteoarticular }\end{array}$ & $\begin{array}{c}\text { lung } \\
\text { septic arthritis }\end{array}$ & $11(40.74)$ & 5 \\
Skin and soft & osteomyelitis & $2(11.11)$ & 1 \\
tissue & wound & $1(3.7)$ & 2 \\
& abdominal wall abscess & $1(3.7)$ & 1 \\
& cervical abscess & $1(3.7)$ & 0 \\
neck cold abscess & $1(3.7)$ & 0 \\
Siver infection & chest wall abscess & $1(3.7)$ & 0 \\
Renleen infection & thigh abscess & $1(3.7)$ & 0 \\
Nenal infection & liver abscess & $2(7.4)$ & 1 \\
& splenic abscess & $1(3.7)$ & 0 \\
\hline
\end{tabular}

Table 2. Secondary clinical foci in the primary diagnostic groups

\begin{tabular}{|c|c|c|c|}
\hline $\begin{array}{l}\text { Primary } \\
\text { diagnosis }\end{array}$ & $\begin{array}{c}\text { Total } \\
\text { number }\end{array}$ & $\begin{array}{l}\text { Number with } \\
\text { secondary foci } \\
\text { (\% of total) }\end{array}$ & Sites secondary foci* \\
\hline Pneumonia & 11 & $2(18.18 \%)$ & 1 liver, 1 prostate \\
\hline Skin \& soft tissue infection & 6 & 0 & - \\
\hline Liver infection & 2 & $2(100 \%)$ & $\begin{array}{c}2 \text { spleen, } 1 \text { prostate, } 1 \text { kidney, } \\
1 \text { peritoneal cavity }\end{array}$ \\
\hline Spleen infection & 1 & $1(100 \%)$ & $\begin{array}{c}1 \text { liver, } 1 \text { sub-diaphragmatic } \\
\text { microabscess }\end{array}$ \\
\hline Osteoarticular infection & 5 & 0 & - \\
\hline
\end{tabular}

*some patients had more than one secondary foci

10 patients had fever for $<1$ month (average 6.7 days). Other presenting complaints were localized pain and swelling (33\%), cough with expectoration $(26 \%)$, generalized weakness $(22 \%)$, wound $(7 \%)$, breathlessness (7\%), and abdominal pain (4\%). Analysis of the clinical manifestations showed 11 (40.74\%) with bacteremia and 16 (59.25\%) without bacteremia. Pneumonia was the most common primary clinical presentation with 11 cases (40.74\%) as shown in Table 1. 9 (33.3\%) patients had an abscess in some part of the body on presentation. Clinical examinations and investigations revealed secondary foci in $5(18.5 \%)$ patients as shown in Table 2. Acute presentations were seen in $21(77.8 \%)$ patients with $<2$ months clinical history and 6 patients had chronic presentations.

3 patients were diagnosed with septic shock on admission. Among the patients with septic shock; one patient had a wound on the foot following a thorn prick since 1 month, 2 patients had pneumonia. 1 patient with septic shock expired on the $8^{\text {th }}$ day of admission. He was a known diabetic with an acute presentation of fever, cough and breathlessness since 3 days. He was diagnosed with lower lobe pneumonia with sepsis in septic shock and type II respiratory failure. On the day of admission blood was sent for culture. Though blood culture report showed B.pseudomallei on the $4^{\text {th }}$ day of admission; he was not started with the recommended regimen.

The prominent risk factors seen were history of type 2 diabetes mellitus, age $>40$ years, alcoholism and smoking. ${ }^{4,8}$ The details are as shown in Table 3.

An analysis of the laboratory investigations showed an average total leucocyte count of 11,556 cells/cumm. Differential count showed marked rise in neutrophils, with $8(29.6 \%)$ patients having 
Table 3. Risk factors for melioidosis

\begin{tabular}{lcc}
\hline Risk factor & Number & Percentage \\
\hline DM & 19 & 70.4 \\
age $>$ 40 Years & 20 & 74.1 \\
exposure to soil & 2 & 7.4 \\
CKD & 1 & 3.7 \\
alcoholism & 4 & 14.8 \\
no defined risk factors & 3 & 11.1 \\
COPD & 2 & 7.4 \\
Smoking & 5 & 18.5 \\
\hline
\end{tabular}

neutrophil percentage of $>90$, and 10 (37.4\%) showing percent neutrophils in the range of 81-90, $4(14.81 \%)$ in the range of $71-80,4(14.81 \%)$ in the range $61-70$ and 1 (3.70\%) in the range of 51-60. Burkholderia pseudomallei was isolated from various samples namely, blood (11 samples), aspirated pus (8), wound swab (2), pleural fluid (2), bronchoalveolar lavage fluid (4), endotracheal aspirate (1), synovial fluid (1), urine (1). In 3 patients B.pseudomallei was isolated from 2 different samples. All isolates were sensitive to imipenem, ceftazidime, cotrimoxazole and amoxicillin-clavulanate. Doxycycline susceptibility was not tested due to non-availability of the method of testing. The turnaround time (TAT) i.e. time from receipt of sample to dispatch of report was on average 5.5 days.

8 patients had undergone ultrasonography (USG) of the abdomen. 4 patients (50\%) had microabscesses in the spleen.

The inpatient case records and outpatient records of the cases were analyzed for the antibiotic treatment, discharge advice and follow up visits. Analysis of the antibiotics treatment given showed that, the treatment regimen given was either i.v. ceftazidime or i.v imipenem/ meropenem for a minimum of 2 weeks and oral cotrimoxazole for a minimum of 12 weeks.

Though $26(96.30 \%)$ had received at least 1 of the antibiotic recommended for melioidosis, only $13(48.15 \%)$ were started with the treatment regimen for melioidosis. $13(48.15 \%)$ completed the intensive treatment regimen. Only 8 (29.63\%) were prescribed the eradication treatment regimen of oral cotrimoxazole for minimum of 12 weeks. 2 patients were not prescribed eradication regimen whereas 3 patients were prescribed for a lesser duration (2 patients were prescribed cotrimoxazole for 2 weeks and 1 patient for 4 weeks). No documented adverse effects were observed in the case records. Out of the 14 patients who were not prescribed the treatment regimen; 1 patient refused admission and one patient expired on the 8th day of admission. 3 patients were discharged within 7 days of admission (out of which 1 patient left against medical advice). One patient was discharged after 5 days of admission, 2 after 6 days. The average TAT of laboratory in reporting B.pseudomallei was 5.5 days (the earliest being 3 days and late being 7 days) and the average duration from admission to diagnosis was 6 days(earliest 4 days and late of 21 days).

Surgical drainage was done in 4 patients with skin and soft tissue abscesses, $20 \mathrm{ml}$ of pus was aspirated from a case of cold abscess in the neck and one patient with a non-healing wound underwent wound debridement. One patient which was inadequately treated came back with reactivation of melioidosis. The patient who was initially admitted for pneumonia came back with hepatic abscess after 3 months of first admission. The second time the patient completed the treatment regimen and recovered.

While one patient with septic shock expired on the $8^{\text {th }}$ day of admission, fourteen patients had prolonged hospital stay ( $>=20$ days).

\section{DISCUSSION}

As we explored the clinical and epidemiological characteristics of cases of melioidosis in our institution, about $70 \%$ of the cases were presented during the monsoon season. In the Western coastal districts of India, the wet season starts with the onset of Southwest monsoon in the month of June and trails off by the end of December with the end of Northeast monsoons. Accordingly, $70 \%$ of cases were seen from July to December. This correlates with literature where an association between monsoon and case occurrence has been recorded. In a similar study from this part of India, $71.6 \%$ patients presented during the heavy Southwest monsoon. ${ }^{8}$ In a 15 year study from Malaysia also the highest frequency of melioidosis admissions were seen during their wet season of November to February. ${ }^{14}$ Heavy rainfall causes the water level on land to rise resulting in aggregation of 
B.pseudomallei on surface soil increases the chances of inhalation of aerosolized bacteria and inoculation through skin abrasions. ${ }^{14,1}$ There are a few contrasting studies which say that exposure to soil has no role in the transmission of the disease. Liu $X$ et al studied 550 melioidosis cases in relation to rainfall and humidity in the highly urbanized city of Singapore. The authors conclude that water is the vehicle for melioidosis transmission. ${ }^{15}$ Some studies have said ingestion of contaminated water as a mode of transmission of B.pseudomallei. ${ }^{16}$

A male preponderance among subjects was noted, likely attributed as males are usually engaged in more outdoor activities. Occupational exposure was not analysed in the study due to lack of records. 2 cases had definite history of exposure; one patient was a case of near drowning a day after which patient developed pneumonia and bacteraemia. One more patient gave history of thorn prick and cellulitis.

Majority of the cases presented to the departments of medicine and surgery (22 cases $81.4 \%)$, but 5 cases (18.5\%) presented to other departments. So, it is very much essential for every medical specialty to be aware of the organism and its varied presentations. Majority of cases $(88.8 \%)$ had at least one risk factor, diabetes being the most common. About two-thirds of the cases occurred in the $>40$ year age group, which in itself is a risk factor. The reason could likely be due to the onset and prevalence of the comorbidities like diabetes mellitus and impaired host immunity in this demographic.

Clinical manifestations in melioidosis varied from acute fulminant septicemia to chronic melioidosis. In this study pneumonia was the most common clinical presentation $40.7 \%$ (11 patients); followed by abscesses (33.3\%) which is similar to other studies. 4 patients had abscess in the internal organs at presentation; out of which 3 patients were found to have abscess in multiple organs. Arthritis and osteomyelitis is also a common presentation with amounting to $18.5 \%$ (5 cases) in this study. Kingsley et.al, studied 67 melioidosis cases and found that 24 (36\%) had pneumonia as the primary clinical manifestation followed by soft tissue abscess in 22 (33\%). ${ }^{17}$ In the study in South India $34.7 \%$ had pulmonary disease, $20 \%$ had musculoskeletal disease and $96.8 \%$ had fever as their chief complaint. ${ }^{8}$ In a fifteen year review from
North Eastern Malaysia the principal presentation in patients was lung infection. In 65 patients out of 158 culture confirmed melioidosis cases had lung infection at presentation followed by skin infection in 44 (27.8\%), osteoarticular infection in 20 (12.7\%) and liver infection in 19 (12.0\%). ${ }^{14}$ In a retrospective study of 57 melioidosis cases by Malczewki et al in Australia the lungs was the most common primary organ involved at presentation $(58 \% ; n=33) .{ }^{18}$

Micro abscesses in the spleen is found to be an important pointer towards melioidosis. Mohan A et al retrospectively studied all children in whom liver and/or spleen abscess was detected on ultrasonography. They found $56 \%$ of children with culture confirmed melioidosis had spleen abscess. Also, 72\% culture negative cases with clinical and imaging features similar to culture proven cases either had serological evidence or improved with empirical antibiotic therapy. ${ }^{19}$ Hence, ultrasonography (USG) of abdomen would be yielding in the diagnosis of suspected melioidosis cases. In our study 6 patients were diagnosed with abscess in the abdominal viscera (in the liver, spleen, prostrate and kidneys). Radiology finding of micro abscess of spleen was seen in $50 \%$ of patients who underwent USG abdomen.

Amongst the laboratory parameters a marked rise in neutrophil count was noted. They form the basis of protective immune response in melioidosis through direct antimicrobial effect and by cytokines production leading to the activation of other immune cells. ${ }^{20}$ TAT of 5.5 days observed in this study could be reduced by training the laboratory staff. In the microbiology laboratory, B.pseudomallei can be easily mistaken for other non-fermenting gram-negative bacilli; as biochemically it broadly resembles other pseudomonads. It takes 24 hours for colonies to appear on solid culture media and nearly 48 hours for colonies to be well-formed. Preliminary tests like gram stain and oxidase test which show bipolar staining and slow oxidase positive in isolates from patients with clinical history like melioidosis should alert the laboratory staff towards the possibility of B.pseudomallei. Additional biochemical tests could be immediately put up. Also alerting the treating team of the possibility followed by a culture report with definitive identification would be important. 
B.pseudomallei has an antibiotic susceptibility pattern which is unique for a gram-negative bacilli. In addition to broad spectrum antibiotics like ceftazidime, carbapenems and cotrimoxazole; it is susceptible to amoxicillin-clavulanic acid. Contrasting to other gram-negative organisms; it has intrinsic resistance towards $1^{\text {st }}$ and $2^{\text {nd }}$ generation cephalosporins and the aminoglycosides. Rare instances of resistance against antibiotics used in treatment of melioidosis is documented in literature. ${ }^{21}$ Though no adverse effects were observed in this study there are studies on larger patient groups which have documented adverse effects. In a study from Australia of the 203 patients who were prescribed trimethoprimsulfamethoxazole for eradication therapy, 61 (30.0\%) experienced adverse effects, which prompted either stoppage, a change in antibiotic or reduction in the of dose cotrimoxazole. ${ }^{22}$ In this study only 13 (48.15\%) were treated adequately. Among the 14 patients in whom the treatment regimen for melioidosis was not initiated, culture reports were available for 10 patients during their hospital stay. From this we can deduce that the treating team was not familiar with B.pseudomallei. Though a column on information regarding the organism and the recommended treatment regimen was observed in the culture reports; prompt verbal communication of the report by the laboratory staff to the treating team would be necessary for melioidosis cases. One patient had reported back with reactivation of melioidosis. In this study, though $48.15 \%$ received the intensive therapy $29.63 \%$ were prescribed the eradication treatment; according to records. As the patients hailed from distant places they would not have come for follow up or would have gone to their nearby health care setup for therapy. These possibilities could not be ascertained. Patients have to be emphasized the importance of a prolonged eradication phase. Relapse of melioidosis can be due to various reasons such as discontinuation of the treatment, inadequate antibiotic treatment protocol due to lack of awareness among health care worker or patient noncompliance, adverse drug reactions and in immune-compromised patients (diabetes, smoking, chronic alcoholism) after completing the adequate treatment regimen. ${ }^{17}$ B. pseudomallei is a slow growing bacteria and may take 48 hours for the colonies to appear on culture media. Hence, by the time it grows and identification is done the patient would be discharged and lost for follow up. In this study 3 patients were discharged within 7 days of admission. The therapeutic regimen must consist of potent bactericidal antimicrobials in the intensive phase and bacteriostatic agents in the eradication phase to curb relapse. ${ }^{23}$ Bacteremia and septic shock are predictors of poor prognosis.

\section{CONCLUSION}

Varied clinical presentation of melioidosis makes the specific clinical diagnosis difficult. Due to the high mortality and morbidity rate, early diagnosis and prompt management is warranted. This requires clinical vigilance and awareness regarding the pathogen among all medical fraternity including the laboratory staff. An alert laboratory team, with an intensive microbiological workup of cultures with suspicion of B.pseudomallei is required for early diagnosis. Lack of adherence to the treatment protocol can lead to reactivation.

\section{ACKNOWLEDGMENTS}

None.

\section{CONFLICT OF INTEREST}

The authors declare that there is no conflict of interest.

\section{AUTHORS' CONTRIBUTION}

All authors listed have made a substantial, direct and intellectual contribution to the work, and approved it for publication.

\section{FUNDING}

None.

\section{DATA AVAILABILITY}

All datasets generated or analyzed during this study are included in the manuscript.

\section{ETHICS STATEMENT}

This article does not contain any experimental studies on human participants or animals performed by any of the authors. It was a retrospective study and only case records of 
patients were accessed and the identity or privacy of the patients were not revealed in the study.

\section{REFERENCES}

1. White NJ. Melioidosis. Lancet. 2003;361(9370):17151722. doi: 10.1016/S0140-6736(03)13374-0

2. Krishnaswami CS. Medical Society Medical meeting, Rangoon, Morphia injector's septicemia. Indian Med Gaz. 1917;52:296-299.

3. Cheng AC, Currie BJ. Melioidosis: epidemiology, pathophysiology, and management. Clin Microbiol Rev. 2005;18(2):383-416. doi: 10.1128/CMR.18.2.383416.2005

4. Limmathurotsakul D, Kanoksil M, Wuthiekanun V, et al. Activities of daily living associated with acquisition of melioidosis in northeast Thailand: a matched casecontrol study. PLoS Negl Trop Dis. 2013;7(2):e2072. doi: 10.1371/journal.pntd.0002072

5. Gassiep I, Armstrong M, Norton R. Human melioidosis. Clin Microbiol Rev. 2020;33(2):e00006-19. doi: 10.1128/CMR.00006-19

6. Yee KC, Lee MK, Chua CT, Puthucheary SD. Melioidosis, the great mimicker: a report of 10 cases from Malaysia. J Trop Med Hyg. 1988;91:249-254.

7. Singh M, Mahmood M. Melioidosis: the great mimicker. J Community Hosp Intern Med Perspect. 2017;7(4):245247. doi: 10.1080/20009666.2017.1348875

8. Vidyalakshmi K, Lipika S, Vishal S, Damodar S, Chakrapani M. Emerging clinico-epidemiological trends in melioidosis: analysis of 95 cases from western coastal India. Int J Infect Dis. 2012;16(7):e491-e497. doi: 10.1016/j.ijid.2012.02.012.

9. Dance D. Treatment and prophylaxis of melioidosis. Int J Antimicrob Agents. 2014;43(4):310-318. doi: 10.1016/j.ijantimicag.2014.01.005

10. Customised Rainfall Information System, Hydromet Division, India meteorological Department Ministry of Earth Sciences. https://hydro.imd.gov.in/ hydrometweb/(S(5uz5lbjb33opgb55jboaut45))/ DistrictRaifall.aspx

11. Quality System Essentials for Antimicrobial Susceptibility Testing of Infrequently Isolated or fastidious Bacteria. In: Methods for Antimicrobial Dilution and Disk Susceptibility Testing of Infrequently Isolated or fastidious bacteria CLSI guideline M45. 3rd ed. Wayne PA: Clinical and Laboratory Standards Institute; 2015:60-63.

12. Cheng AC, Fisher DA, Anstey NM, Stephens DP, Jacups SP, Currie BJ. Outcomes of patients with melioidosis treated with meropenem. Antimicrob Agents Chemother. 2004;48(5):1763-1765. doi:

\subsection{8/AAC.48.5.1763-1765.2004}

13. Lipsitz R, Garges S, Aurigemm R, et al. Workshop on treatment of and postexposure prophylaxis for Burkholderia pseudomallei and B. mallei infection, 2010. Emerg Infect Dis. 2012;18(12):e2. doi: 10.3201/ eid1812.120638

14. Zeuter AR, Yean CY, Abumarzouq M, Rahman ZA, Deris ZZ, Harun A. The epidemiology and clinical spectrum of melioidosis in a teaching hospital in a North-Eastern state of Malaysia: a fifteen year review. BMC Infectious Diseases 2016;16:333. doi: 10.1186/s12879-016-15832

15. Liu X, Pang L, Sim SH, et al. Association of Melioidosis Incidence with Rainfall and Humidity, Singapore, 2003-2012. Emerg Infect Dis. 2015;21(1):159-162. doi: 10.3201/eid2101.140042

16. Inglis TJJ, Garrow SC, Henderson M, et al. Burkholderia pseudomallei Traced to Water Treatment Plant in Australia. Emerg Infect Dis. 2000;6(1):56-59. doi: 10.3201/eid0601.000110

17. Kingsley PV, Leader M, Nagodawithana NS, Tipre M, Sathiakumar N. Melioidosis in Malaysia: A Review of Case Reports. PLoS Negl Trop Dis. 2016;10(12):e0005182. doi: 10.1371/journal. pntd. 0005182

18. Malczewski AB, Oman KM, Norton RE, Ketheesan N. Clinical presentation of melioidosis in Queensland, Australia. Trans R Soc Trop Med Hyg. 2005;99(11):856860. doi: 10.1016/j.trstmh.2005.06.015

19. Mohan A, Manan K, Tan LS, et al. Detection of spleen abscesses facilitates diagnosis of melioidosis in Malaysian children. Int J Infect Dis. 2020;98:59-66. doi: 10.1016/j.ijid.2020.06.025

20. Easton A, Haque A, Chu K, Lukaszewski R, Bancroft GJ. A Critical Role for Neutrophils in Resistance to Experimental Infection with Burkholderia pseudomallei. J Infect Dis. 2007;195(1):99-107. doi: 10.1086/509810

21. Dance DAB, Wuthiekanun V, Chaowagul W, White NJ. The antimicrobial susceptibility of Pseudomonas pseudomallei. Emergence of resistance in vitro and during treatment. J Antimicrob Chemother. 1989;24(3):295-309. doi: 10.1093/jac/24.3.295

22. Sullivan RP, Ward L, Currie BJ. Oral eradication therapy for melioidosis: Important but not without risks. Int J Infect Dis. 2019;80:111-114. doi: 10.1016/j. ijid.2019.01.019

23. Pitman MC, Luck T, Marshall CS, Anstey NM, Ward L, Currie BJ. Intravenous Therapy Duration and Outcomes in Melioidosis: A New Treatment Paradigm. PLoS Negl Trop Dis. 2015;9(3):e0003586. doi: 10.1371/journal. pntd.0003586 